Document downloaded from:

http://hdl.handle.net/10251/68263

This paper must be cited as:

Uris Martínez, A.; Gómez Lozano, V.; Candelas Valiente, P.; Belmar Ibáñez, F. (2014). On the Role of the Geometrical Parameters in the Ultrasonic Transmission Through Plates with Subwavelength Holes Arrays. Acta Acustica united with Acustica. 100(4):595-603. doi:10.3813/AAA.918739.

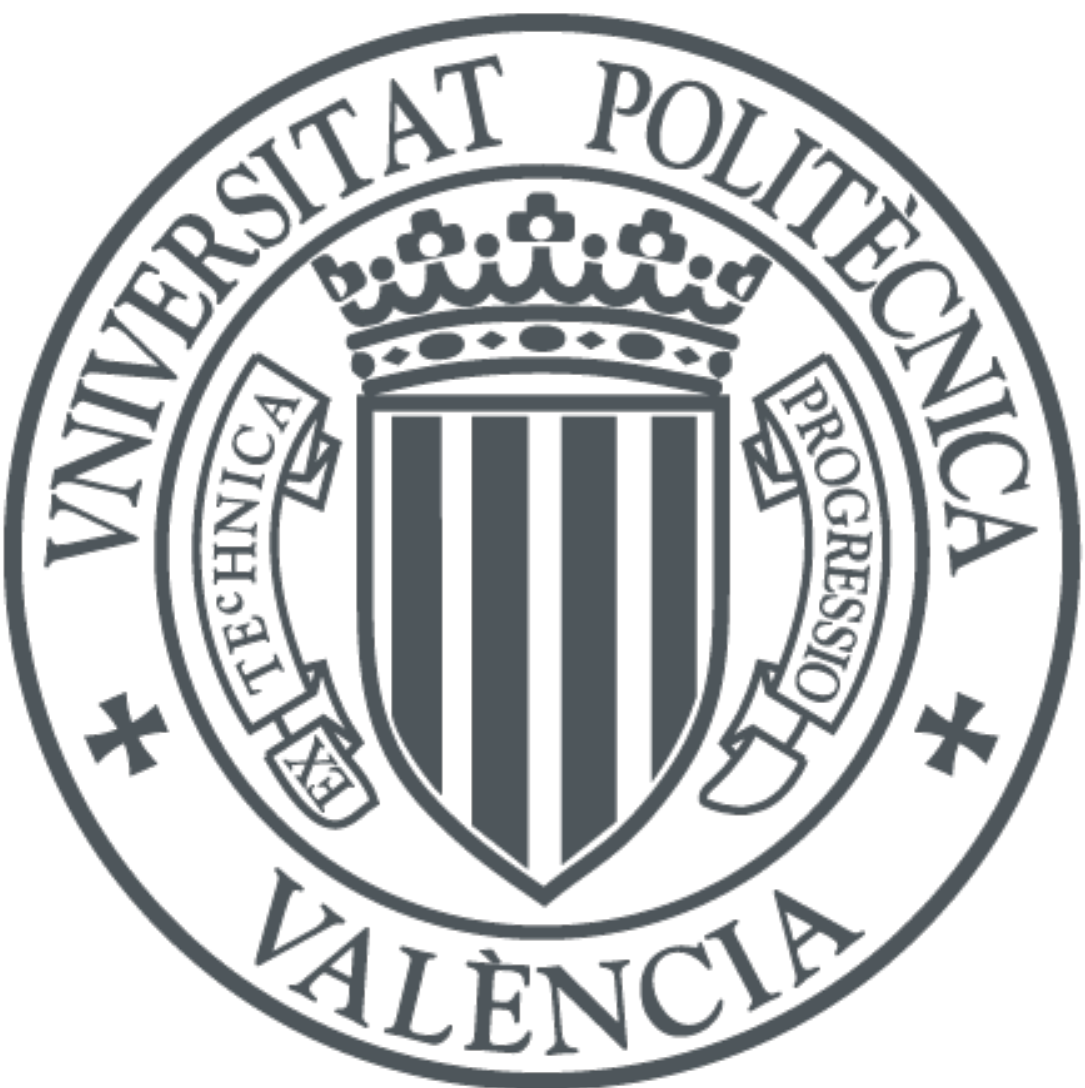

The final publication is available at

http://dx.doi.org/10.3813/AAA.918739

Copyright Hirzel Verlag (S. Hirzel Verlag)

Additional Information 


\title{
ON THE ROLE OF THE GEOMETRICAL PARAMETERS IN THE ULTRASONIC TRANSMISSION THROUGH PLATES WITH SUBWAVELENGTH HOLES ARRAYS
}

\author{
Antonio Uris*, Vicente Gomez-Lozano, Pilar Candelas, Francisco Belmar \\ Centro de Tecnologías Físicas: Acústica, Materiales y Astrofísica. División Acústica. \\ Universitat Politécnica de Valencia. Camino de Vera s/n. 46022 Valencia, Spain. \\ Phone: 34.963877528; Fax: 34.963879525; E-mail: auris@fis.upv.es \\ *Corresponding author.
}

\begin{abstract}
This paper presents an overview of the recents studies on the ultrasonic transmission through subwavelength holes arrays. The role of the geometrical parameters of the perforated plates in the transmission features has been reported by using a theoretical model under the rigid-solid assumption. It is shown that the transmission spectrum can be tailored by varying the geometrical parameters.
\end{abstract}

PACS no. 43.35. +d, 42.79. Dj 


\section{INTRODUCTION}

The interaction of waves with periodic structures dates from, at least, the end of the XIX century with the study of sound reflection of one-dimensional gratings by Rayleigh [1]. Since then numerous authors have studied the problem. The behaviour of electromagnetic and mechanical waves in periodic structures was a subject of a book by Brillouin [2], that he tried on unifying concepts about both electromagnetic and mechanical waves. From the knowledge of the wave nature of electromagnetic and acoustic waves, some of the ideas developed for electromagnetic waves have been transferred to acoustics taking into account their differences: the electromagnetic wave is a vector transverse wave while acoustic wave is a scalar longitudinal wave. The concept of sonic crystal for acoustics is based on the idea of photonic crystal for electromagnetic waves [3].

In 1998, Ebbesen et al. [4] observed enhanced transmission of light through periodic arrays of subwavelength holled drilled on a metal film. They observed that the light transmission per hole at specific frequencies, correlated with the periodicity, is much larger that predicted by Bethe's theory [5]. Since then, extraordinary optical transmission through metallic films perforated with subwavelength apertures has atracted much attention and has been subject of numerous theoretical and experimental studies. A recent review by Garcia-Vidal et al. [6] describes the physical mechanisms and the developments in the transmission of light through subwavelength apertures up to 2010.

As well as the case of photonic and sonic crystals, the idea of optical transmission through subwavelength apertures has been transferred to acoustics to obtain effects similar to extraordinary optical transmission. In the last six years, this area of study has received a lot of attention. Lu et al. [7] reported experimentally the so-called extraordinary acoustic 
transmission through a subwavelength slit array. Zhou and Kriegsman [8] predicted complete sound transmission through subwavelength hole arrays by means of the scattering matrix technique and Hou et al. [9] reported experimentally the acoustic transmission through subwavelength holes arrays. The main contribution to the transmission peaks are the Fabry-Perot resonances inside the holes. Christensen et al. [10] reported theoretical results in the rigid solid limit for subwavelength slits and square holes arrays. Estrada et al. [11] have shown both theoretically and experimentally in the ultrasonic range, that, at certain frequencies, perforated plates with subwavelength holes arrays exhibit higher attenuation that predicted by mass law apart from the extraordinary acoustic transmission. This effect is due to the Wood anomaly [12]. Estrada et al. [13] also demonstrated that the ultrasonic transmission properties of a perforated plate with subwavelength holes arrays and inmersed in water, is an interplay between Fabry-Perot resonances inside the holes, lattice resonances in periodic arrays and elastic Lamb modes. Wang $[14,15]$ uses acoustic impedances to describe the extraordinary acoustic transmission by assuming piston-like behaviour at the holes apertures, and revealed that the singularity of the radiation impedance of the holes array is the origin of the full transmission. A suppresion of the full transmission peak can be achieved when the hole array basis is asymmetric [16]. The acoustic transmission through plates perforated with a quasiperiodic arrays of subwalength apertures has been studied by Hao et al. [17].

The role of the geometrical parameters of the hole array is important in the ultrasonic transmission through perforated plates and has been subject of several studies [18-22]. The purpose of this paper is to describe and summarize theoretically the set of geometrical parameters that affect the ultrasonic transmission through subwavelength holes arrays. A rigid solid model is used to calculate ultrasound transmission. The calculations with the 
rigid solid model predicts well the transmission dips and full transmission peaks, but there are some transmission peaks, specially at oblique incidence, that the rigid solid model can not predict. These discrepancies can be attributed to the assumption made about the behaviour of the solid part of the device, namely zero energy transmission across the solid part of the plate. The parameter that quantifies the transmission of acoustic energy between semi-infinites media is the impedance ratio. A high contrast of impedances between the solid and the fluid, involves minor discrepancies between rigid solid model calculations and measurements. This is easily understood because a perfect match of impedances mean a perfect fluid-solid coupling and a full transmission of energy between fluid and solid, whereas a perfect rigid solid implies an infinite contrast of impedances. If only normal transmission coefficient is considered, the differences between rigid solid model and measurement data, as expected, are lower because the wave vector has no component in the plate. A very different situation occurs when we take into account oblique incidence. In this case, measurements depicts a scenario when new peaks of transmission appear and a complex interplay between maxima and minima occurs, this makes clear that the limitations of the rigid solid model [13].

\section{BASIC THEORY. RIGID SOLID MODEL.}

Consider a plate of thickness $t$ which is perforated with $P$ cylindrical holes of radius $r_{i}^{0}$ in positions determined by their centres $\mathbf{r}_{i}$, as schematically shown in Figure 1. For the study of the pressure field, we split the space in three regions, namely incident region [I], holes in plate region [II] and radiation region [III].

Assuming an incident plane wave $e^{i\left(\mathbf{k}_{0} \cdot \mathbf{r}-\omega t\right)}$ impinging in the plate, the pressure field in the incidence region (region $[\mathrm{I}]$ in the Figure 1) is the sum of the incident and the scattered 
wave, and can be written, dropping the time dependence $e^{-i \omega t}$, in terms of a series of planes waves, in the form [23]

$$
\phi_{I}(\mathbf{r})=e^{i\left(\mathbf{Q}_{0} \cdot \mathbf{r}_{\|}-q_{0} z\right)}+\iint \beta^{+}(\mathbf{Q}) e^{i\left(\mathbf{Q} \cdot \mathbf{r}_{\|}+q z\right)} d^{2} Q
$$

where $\mathbf{k}_{0}=\left(\mathbf{Q}_{0}, q_{0}\right)$ is the wave vector, $k_{0}=\omega / c, q=\sqrt{k_{0}^{2}-Q^{2}}$.

The pressure field inside the holes (region [II] in the Figure 1) is expanded as a traveling wave in the $\mathrm{z}$ direction and a standing wave in the transverse one. Using cylindrical waves to take advantage of the symmetry of the problem, and yields

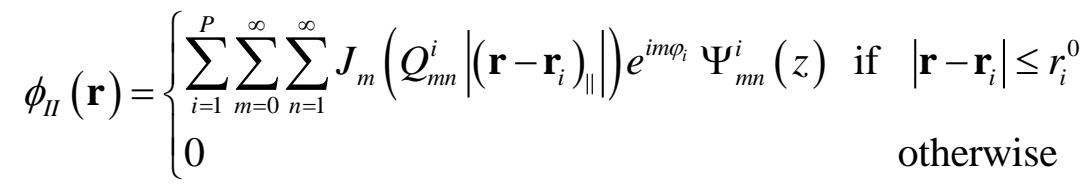

where $q_{m n}^{i}=\sqrt{k_{0}^{2}-Q_{m n}^{i 2}}, J_{m}(\ldots)$ is the Bessel function of the first kind and order $m$ and $\Psi_{m n}^{i}(z)$ is defined as $\Psi_{m n}^{i}(z)=\alpha_{m n}^{i+} e^{i q_{m n}^{i} z}+\alpha_{m n}^{i-} e^{-i q_{m n}^{i} z}$

In the radiation region (region [III] in the Figure 1), the pressure field is expanded in plane waves and can be written as

$$
\phi_{I I I}(\mathbf{r})=\iint \beta^{-}(\mathbf{Q}) e^{i\left(\mathbf{Q} \cdot \mathbf{r}_{\|}-q(z+h)\right)} d^{2} Q
$$

The $P$ holes are periodically distributed throughout the whole plate, and can be considered as a lattice basis with a unit cell area $S$ and defined by the vectors $\left(\mathbf{a}_{1}, \mathbf{a}_{2}\right)$. In this way, the coefficients $\beta^{ \pm}(\mathbf{Q})$ can be expanded in Fourier series [24], giving the following discrete pressure field equations for regions [I] and [III]

$$
\begin{gathered}
\phi_{I}(\mathbf{r})=2 e^{i\left(\mathbf{Q}_{0} \cdot \mathbf{r}_{\|}\right)}+\sum_{\mathbf{G}} \beta_{\mathbf{G}}^{+} e^{i\left(\mathbf{Q}_{\mathbf{G}} \cdot \mathbf{r}_{\|}+q_{\mathbf{G}} z\right)} \\
\phi_{I I I}(\mathbf{r})=\sum_{\mathbf{G}} \beta_{\mathbf{G}}^{-} e^{i\left(\mathbf{Q}_{\mathbf{G}} \cdot \mathbf{r}_{\|}+q_{\mathbf{G}}(z+h)\right)}
\end{gathered}
$$

where $\mathbf{Q}_{\mathbf{G}}=\mathbf{Q}_{0}+\mathbf{G}, \mathbf{G}$ is the reciprocal lattice vector, and $q_{\mathbf{G}}=\sqrt{k_{0}^{2}-Q_{\mathbf{G}}{ }^{2}}$. 
If we model the plate as a rigid solid (RSM), then the sound field inside the solid vanishes, and the polar eigenfunctions inside each hole must satisfy $J_{m}^{\prime}\left(Q_{m n}^{i} r_{i}^{0}\right)=0$, that is, zero normal velocity at the hole walls.

To determine the coefficients $\beta_{\mathrm{G}}^{ \pm}$and $\alpha_{\mathbf{G}}^{ \pm}$in the equations (2),(4) and (5), we impose the continuity of the pressure and normal velocity in the interfaces holes between the regions $[\mathrm{I}]$ and [II], i.e. at $z=0$ and the regions[II] and [III], $(z=-h)$.

Taking advantage of the orthogonality of the exponentials and Bessel functions on the unit cell, we obtain [23]

$$
\begin{gathered}
2 \overline{I_{m^{\prime} n^{\prime}}^{j}\left(\mathbf{Q}_{0}\right)}=-\frac{1}{S} \sum_{i=1}^{P} \sum_{m=0}^{\infty} \sum_{n=1}^{\infty} q_{m n}^{i} \Psi_{m n}^{\prime i}(0) M_{m m^{\prime} n n^{\prime}}^{i j}\left(\mathbf{Q}_{\mathbf{G}}\right)+\sum_{i=1}^{P} \sum_{m=0}^{\infty} \sum_{n=1}^{\infty} 2 \pi \delta_{i j} \delta_{m m^{\prime}} \delta_{n n^{\prime}} P_{m n}^{i} \Psi_{m n}^{i}(0)(5) \\
0=\frac{1}{S} \sum_{i=1}^{P} \sum_{m=0}^{\infty} \sum_{n=1}^{\infty} q_{m n}^{i} \Psi_{m n}^{\prime i}(-h) M_{m m^{\prime} n n^{\prime}}^{i j}\left(\mathbf{Q}_{\mathbf{G}}\right)+\sum_{i=1}^{P} \sum_{m=0}^{\infty} \sum_{n=1}^{\infty} 2 \pi \delta_{i j} \delta_{m m^{\prime}} \delta_{n n^{\prime}} P_{m n}^{i} \Psi_{m n}^{i}(-h)
\end{gathered}
$$

where the coefficients in the above equations are defined by means of the formulas (8), (9), (10) and (11)

$$
\begin{gathered}
M_{m m^{\prime} n n^{\prime}}^{i j}\left(\mathbf{Q}_{\mathbf{G}}\right)=\sum_{\mathbf{G}} \frac{I_{m n}^{i}\left(\mathbf{Q}_{\mathbf{G}}\right) \overline{I_{m^{\prime} n^{\prime}}^{j}\left(\mathbf{Q}_{\mathbf{G}}\right)}}{q_{\mathbf{G}}} \\
\left.P_{m n}^{i}=\int_{0}^{r_{i}^{0}} J_{m}^{2}\left(Q_{m n}^{i} \mid\left(\mathbf{r}-\mathbf{r}_{j}\right)_{\|}\right)\right) r d r \\
I_{m n}^{i}\left(\mathbf{Q}_{\mathbf{G}}\right)=e^{-i \mathbf{Q}_{\mathbf{G}} \cdot \mathbf{r}_{i}} \int_{0}^{2 \pi} \int_{0}^{r_{i}^{0}} J_{m}\left(Q_{m n}^{i} r\right) e^{i m \varphi} e^{-i \mathbf{Q}_{\mathbf{G}} \cdot \mathbf{r}} r d r d \varphi \\
\Psi_{m n}^{\prime i}(z)=\alpha_{m n}^{i+} e^{i q_{m n} z}-\alpha_{m n}^{i-} e^{-i q_{m n} z}=\frac{i}{q_{m n}^{i}} \frac{\partial \Psi_{m n}^{i}}{\partial z}
\end{gathered}
$$

and the $\beta_{\mathbf{G}}^{ \pm}$coefficients are related to the $\alpha_{\mathbf{G}}^{ \pm}$by means of

$$
\beta_{\mathbf{G}}^{+}=\frac{1}{S} \sum_{i=1}^{P} \sum_{m=0}^{\infty} \sum_{n=1}^{\infty} \frac{q_{m n}^{i}}{q_{\mathbf{G}}} I_{m n}^{i}\left(\mathbf{Q}_{\mathbf{G}}\right) \Psi_{m n}^{\prime i}(0)
$$




$$
\beta_{\mathbf{G}}^{-}=-\frac{1}{S} \sum_{i=1}^{P} \sum_{m=0}^{\infty} \sum_{n=1}^{\infty} \frac{q_{m n}^{i}}{q_{\mathbf{G}}} I_{m n}^{i}\left(\mathbf{Q}_{\mathbf{G}}\right) \Psi_{m n}^{\prime i}(-h)
$$

Truncating the series (6) and (7) in $m=n=M$ and rearranging, we obtain the following system of $2 \times M \times M \times P$ equations in the variables $\alpha_{m n}^{i \pm}$ witch can be solved, for each pair frequency $\omega$ and angle of incidence $\mathbf{Q}_{0}$, by means of standard procedures

$$
\begin{aligned}
& \sum_{i=1}^{P} \sum_{m=1}^{M} \sum_{n=1}^{M}\left(A_{m n^{\prime} n n^{\prime}}^{i j} \cdot \alpha_{m n}^{i+}+B_{m m^{\prime} n n^{\prime}}^{i j} \cdot \alpha_{m n}^{i-}\right)=2 \cdot \overline{I_{m^{\prime} n^{\prime}}^{j}\left(\mathbf{Q}_{0}\right)} \\
& \sum_{i=1}^{P} \sum_{m=1}^{M} \sum_{n=1}^{M}\left(C_{m m^{\prime} n n^{\prime}}^{i j} \cdot \alpha_{m n}^{i+}+D_{m m^{\prime} n n^{\prime}}^{i j} \cdot \alpha_{m n}^{i-}\right)=0
\end{aligned}
$$

where the matrices A,B,C and D are defined by

$$
\begin{aligned}
& A_{m m^{\prime} n n^{\prime}}^{i j}=2 \pi \delta_{i j} \delta_{m m^{\prime}} \delta_{n n^{\prime}}{ }_{m n n}^{i}-\frac{1}{S} q_{m n}^{i} M_{m m^{\prime} n n^{\prime}}^{i j} \\
& B_{m n^{\prime} n^{\prime}}^{i j}=2 \pi \delta_{i j} \delta_{m m^{\prime}} \delta_{n n^{\prime}} P_{m n}^{i}+\frac{1}{S} q_{m n}^{i} M_{m m^{\prime} n n^{\prime}}^{i j} \\
& C_{m m^{\prime} n n^{\prime}}^{i j}=B_{m m^{\prime} n n^{\prime}}^{i j} \cdot e^{-i q_{m n^{i}}^{i} h} \\
& D_{m m^{\prime} n^{\prime}}^{i j}=A_{m m^{\prime} n n^{\prime}}^{i j} \cdot e^{i q_{m n^{i}}^{i} h}
\end{aligned}
$$

At this point, the coefficients $\beta_{\mathrm{G}}^{ \pm}$can be obtained evaluating back the eqs. (12) and (13).

The ultrasound transmission coefficient is then calculated from the ultrasound power radiated by an infinite plate [25]

$$
\Pi(\omega)=\frac{\rho c k_{0}}{8 \pi^{2}} \operatorname{Re}\left\{\iint_{-\infty}^{\infty} \frac{|V(\mathbf{Q})|^{2}}{\sqrt{k_{0}^{2}-Q^{2}}} d^{2} Q\right\} \quad V(\mathbf{Q})=\mathscr{F}_{x} \mathscr{T}_{y}\left\{\left.\frac{i \partial_{z} p}{\omega \rho}\right|_{z=0, z=-h}\right\}
$$

where $\mathscr{F}_{x} \mathscr{F}_{y}$ means double spatial Fourier transform. This formula applied to our case yields

$$
\tau=\frac{\Pi_{T}(\omega)}{\Pi_{0}(\omega, \theta, \varphi)}=\sum_{\mathbf{G}} \operatorname{Re}\left\{\frac{q_{\mathbf{G}}}{q_{0}}\right\}\left|\beta_{\mathbf{G}}^{-}\right|^{2}
$$

\section{RESULTS AND DISCUSSION}


When an ultrasonic wave impinges onto a subwavelength perforated plate, a typical transmission spectrum is obtained. Figure 2(a) shows calculated transmitted ultrasound power coefficient as a function of frequency, $f$, in water at normal incidence for a plate drilled with square lattice subwavelength holes arrays of diameter $\mathrm{d}=2 \mathrm{~mm}$, lattice period $\mathrm{p}=4 \mathrm{~mm}$ and plate thickness $\mathrm{t}=2 \mathrm{~mm}$. It can be clearly seen a pronounced transmission peak at $250 \mathrm{kHz}$ followed by a minimun transmission value. The transmission peak is related to the Fabry-Perot resonances inside the holes while the minimum transmission is just the Wood anomaly similar to those observed in optical gratings [12]. Wood anomalies are an effect observed in the spectrum of ligth when ligth impinges in optical diffraction gratings. It was observed rapid variations in the intensity of specific diffracted spectral orders in narrow frequency bands. The minimum is produced by a coherent interference.

The Wood anomaly for normal incidence is given by $\frac{\omega}{c}=\sqrt{\left(\frac{2 \pi m}{p}\right)^{2}+\left(\frac{2 \pi n}{p}\right)^{2}}$, where $p$ is the lattice period, $m, n$ are called Miller indices and $c$ is the speed of ultrasound in water. The positions of the Wood anomaly are $370 \mathrm{kHz}$ and $520 \mathrm{kHz}$ for $(\mathrm{m}, \mathrm{n})=(1,0)$ and $(1,1)$, respectively. Figure 2(b) shows calculated transmitted ultrasound power coefficient as a function of both the frequency and the incidence angle. It is seen the strong angle dependence of the Wood anomalies. The transmission peaks also exhibit angledependent behaviour.

\subsection{Influence of plate thickness}

It is seen from the open tube Fabry-Perot resonant condition $t=n \frac{\lambda}{2}$, where $\lambda$ is the wavelength and $\mathrm{n} \in \mathrm{N}$, that the resonant transmission peaks are directly related to the plate thickness, therefore when the plate thickness increases, more transmission maxima will 
appear and they will move toward lower frequencies. Fig. 3 shows calculated transmitted ultrasound power coefficient as a function of frequency, $f$, in water at normal incidence for a plate drilled with subwavelength holes arrays of diameter $\mathrm{d}=2 \mathrm{~mm}$, square lattice period $\mathrm{p}=4 \mathrm{~mm}$ and for four different values of the plate thickness, $\mathrm{t}=2,3,4$, and 5 . It is seen that as thickness increases from 2 to $3 \mathrm{~mm}$ the second order Fabry-Perot resonance $(\mathrm{n}=2)$ appears at $330 \mathrm{kHz}$. The position of the peaks do not agree precisely with those calculated with the open tube Fabry-Perot condition due to it is highly influenced by the array periodicity. When the plate thickness is $5 \mathrm{~mm}$ the second, third and fourth order resonances are visualized. Due to the lattice period is the same for all the plate thickness the positions of the Wood anomalies $(370 \mathrm{kHz}$ and $520 \mathrm{kHz})$ do not change.

\subsection{Influence of hole filling fraction}

The hole filling fraction is a parameter that has a large impact on the ultrasonic transmission through perforated plates [18]. For a square array, the hole filling fraction,

$f f$, is given by $\frac{\pi d^{2}}{4 p^{2}}$, where $d$ is the hole diameter and $p$ is the lattice period. Thus, the hole filling fraction can be modified by varying the diameter or the lattice period. Calculated transmitted ultrasound power coefficient as a function of frequency, $f$, in water at normal incidence for a plate drilled with subwavelength holes arrays of diameter $d=3$ $\mathrm{mm}$, plate thickness $\mathrm{t}=4 \mathrm{~mm}$ and for three square lattice periods $\mathrm{p}=4,6$ and $8 \mathrm{~mm}$, is shown in Figure 4. As the filling fraction decreases, more transmission dips show up resulting from Wood anomaly and these dips move toward lower frequencies. The position of the transmission peaks related to the Fabry-Perot resonances are influenced by displacement of the Wood anomalies. It can also be observed that the transmission 
peaks become narrower as the filling fractions decrease, as in the case of diffraction gratings [26].

Figure 5 shows the calculated results for a plate of thickness $t=4 \mathrm{~mm}$, square lattice period $\mathrm{p}=6 \mathrm{~mm}$ and for three holes diameters $\mathrm{d}=2,3$ and $4 \mathrm{~mm}$. In this case, as the lattice period is constant, the positions of transmission dips corresponding to the Wood anomaly remains at the same frequencies when the filling fraction varies. The transmission peaks related to the Fabry-Perot resonances become narrower as the filling fractions decrease just like results obtained by varying lattice periods.

\subsection{Influence of lattice geometry}

A periodical repetition of $N$ holes throughout the plate can be considered as a lattice basis with unit-cell area $\mathrm{S}$ and defined by the primitive vectors $(\vec{a}, \vec{b})$. For normal incidence, the Wood anomaly condition is given by $\frac{\omega}{c}=|\mathbf{G}|$, where $\mathbf{G}=m \mathbf{a}_{1}+n \mathbf{a}_{2}$, is the reciprocal lattice vector and $m, n$ are the Miller indices. For a square array the primitive vectors are $\mathbf{a}_{1}=\frac{2 \pi}{p} \mathbf{x}$ and $\mathbf{a}_{2}=\frac{2 \pi}{p} \mathbf{y}$, and for a triangular array the primitive vectors are $\mathbf{a}_{1}=\frac{2 \pi}{p}\left(\mathbf{x}+\frac{1}{\sqrt{3}} \mathbf{y}\right)$ and $\mathbf{a}_{2}=\frac{2 \pi}{p}\left(\mathbf{x}-\frac{1}{\sqrt{3}} \mathbf{y}\right)$. Therefore, the frequencies at which the transmission dips related with Wood anomaly occur are directly related with the lattice geometry. Figures $6(\mathrm{a}-\mathrm{c})$ show the calculated results for a plate of thickness $t=$ $4 \mathrm{~mm}$, hole diameter $\mathrm{d}=2 \mathrm{~mm}$ and for three different lattice periods $\mathrm{p}=4,5$ and $6 \mathrm{~mm}$, respectively arranged with both square and triangular lattice hole arrays. Comparison between results obtained for both square and triangular lattices reveals that the 
transmission dips corresponding to Wood anomalies appear at lower frequencies in the square lattices [19]. As the filling factor of the square lattice is lower than triangular one, the transmission peaks of the square lattice geometries are narrower than the triangular ones.

\subsection{Two periodic hole arrays}

Two periodic holes arrays arrangement consists in a primary square array which is decorated with a larger secondary array that can be square or rectangular. The symmetry of the resulting structure is given by the symmetry of the secondary array and consists in a cell with many holes and lattice constant large enough to include all the holes. The transmission properties of a plate perforated with the so called double periodicity can be explored by considering a rectangular compound holes array as described in Figure 7. The compound array is drilled on a plate of thickness $\mathrm{t}=4 \mathrm{~mm}$. The lattice is rectangular with constants $\mathrm{a}_{1}=16 \mathrm{~mm}$ and $\mathrm{a}_{2}=4 \mathrm{~mm}$ and the basis consists in nine holes, eigth of them of diameter $d_{1}=1 \mathrm{~mm}$ and equally spaced, and the ninth, with a diameter $d_{2}=3 \mathrm{~mm}$, drilled in the centre of the array. This array of holes can be regarded as a superposition of two arrays. The primary array is a rectangular distribution of holes having diameter $\mathrm{d}_{2}=3$ $\mathrm{mm}$ and rectangular unit cell of dimensions $\mathrm{a}_{1}=16 \mathrm{~mm}$ and $\mathrm{a}_{2}=8 \mathrm{~mm}$ whereas the secondary array is a square distribution of holes having diameter $\mathrm{d}_{1}=1 \mathrm{~mm}$ and a unit cell period of $\mathrm{a}_{2} / 2=4 \mathrm{~mm}$. Figures $8(\mathrm{a})$ and $8(\mathrm{~b})$ show the calculated transmitted ultrasound power coefficient as a function of frequency, $f$, in water for the primary and secondary array respectively. Although the plate thickness is the same, the position of the transmission peaks related to Fabry-Perot resonances shift considerably by the effect of the hole size and the period [19]. The transmission of the plate with two periodic holes arrays is depicted in Figure 8(c). It could be seen that the transmission spectrum is simply the 
addition of the primary and secondary array spectrum with the transmission peaks at the same frequencies than the simple arrays [20], and it is in this sense that it can speak of double periodicity.

\subsection{Multiple-sublattice periodic hole arrays}

When two or more holes are arranged in a unit cell, the resulting structure is a multiplesublattice periodic holes array. With increasing the number of holes per unit cell and varying the spacing between holes, the number of resonances will increased and the transmission spectrum will change. The question of the interaction between different resonances is answered by considering $4 \times 4$ multiple-sublattice holes arrays placed in water. In each $4 \times 4$ squared holes arrays considered, the plate thickness is $t=3 \mathrm{~mm}$, the period of the unit cell is $\mathrm{a}=5 \mathrm{~mm}$, the hole diameter $\mathrm{d}=0.7 \mathrm{~mm}$ and three values of inter-hole distances within the unit cell, $p$, are considered. The values of $p$ considered are $\mathrm{a} / 4, \mathrm{a} / 5$ and a/6. Figure 9 shows the multiple-sublattice hole unit cells considered. Calculated transmitted ultrasound power coefficient as a function of frequency, $f$, in water at normal incidence of the three $4 \times 4$ multiple-sublattice holes arrays considered, are showed separately in Figures 10 (a-c) for the samples with the inter-hole distance a/4, a/5 and a/6, respectively. When the inter-hole distance within the unit cell is a/4 (Fig. 10a), the resulting sample is a square lattice holes array with period a/4. The transmission peaks correspond to the Fabry-Perot resonances, and transmission dip at the frequency around $1180 \mathrm{kHz}$ correspond to the Wood anomaly. By reducing the inter-hole distance to a/5 (Fig. 10b), new transmission dips resulting from Wood anomaly corresponding to the period of the unit cell appear. It is also observed that the amplitude of the first order Fabry-Perot resonance peak is invariable but the amplitude of the second and higher orders is reduced. A remarkable feature is that the first order Fabry-Perot resonance 
splits into two peaks. When the inter-hole distance is a/6, the amplitude reduction of the second and higher orders Fabry-Perot resonance peaks is more important, and the splitting of the first order Fabry-Perot resonance peak remains invariable. The splitting arises from the interference between holes $[16,21]$. When the phase difference between holes in a unit cell approaches $\pi$, the interference is destructive. Whether the inter-hole distance reduces within a unit cell, the transmission spectrum will tend to become a spectrum with only one hole per unit cell [21].

\subsection{Fractal-featured hole arrays}

Previously, only periodic distribution of holes had been addressed. An example of aperiodic structures is the fractal one. A well known fractal structure is the Sierpinski Carpet, which construction follows an iterative process. The effect of the geometry selfsimilarity on the transmission spectrum is analyzed using three iterative generations of the Sierpinsky Carpet. A square distribution of holes having diameter $0.5 \mathrm{~mm}$ was used as a first step and a unit cell period $\mathrm{a}=1.5 \mathrm{~mm}$. The second iterative generation was made with a square distribution of holes having diameters $0.5 \mathrm{~mm}$ and $1.5 \mathrm{~mm}$, and a unit cell period $\mathrm{a}=4.5 \mathrm{~mm}$. The third and last iterative generation was made with a square distribution of holes having diameters $0.5 \mathrm{~mm}, 1.5 \mathrm{~mm}$ and $4.5 \mathrm{~mm}$, and a unit cell period $\mathrm{a}=13.5 \mathrm{~mm}$. Figures $11(\mathrm{a}-\mathrm{c})$ show a schematic diagram of the iterative generations considered. In all samples considered, the plate thickness was $4 \mathrm{~mm}$. Figures 12(a-c) show the calculated transmitted ultrasound power coefficient as a function of frequency, $f$, in water at normal incidence for each of the three iterations considered. The transmission spectrum of the square distribution of holes with diameter $\mathrm{d}=0.5 \mathrm{~mm}$ and a unit cell period $\mathrm{a}=1.5 \mathrm{~mm}$ is depicted in Figure 12(a). The transmission peaks observed correspond to the Fabry-Perot resonances. The transmission dip corresponding 
to Wood anomaly is out of frequency range (around $980 \mathrm{kHz}$ ). The transmission spectrum of the second iterative generation, made with holes having diameters $0.5 \mathrm{~mm}$ and $1.5 \mathrm{~mm}$ and unit cell period of $4.5 \mathrm{~mm}$ is showed in Figure 12(b). The resulting structure is an arrangement that consists in two periodic holes arrays having a primary square array which is decorated with a larger secondary square array. The transmission spectrum is the addition of the primary and secondary array spectrum. One remarkable feature if the existence of a transmission dip at $165 \mathrm{kHz}$ due to a destructive interference between the holes of the primary and secondary array $[16,21]$. The third iteration made with holes having diameters $0.5 \mathrm{~mm}, 1.5 \mathrm{~mm}$ and $4.5 \mathrm{~mm}$, and a unit cell period $\mathrm{a}=$ $13.5 \mathrm{~mm}$, is depicted in Figure 12(c). Like in the second iteration case, the transmission spectrum has the characteristics peaks and dips of each contributed array.

\subsection{Effect of lattice vacancies}

Perforated plates with subwavelength holes arrays containing holes vacancies in their unit cells show particular ultrasound transmission properties. The effect of the holes vacancies on the ultrasound transmission spectrum is analyzed using $3 \times 3$ squared holes arrays placed in water. The holes having diameter $3 \mathrm{~mm}$ were drilled on a plate of thickness $t=2 \mathrm{~mm}$. A unit cell period of $15 \mathrm{~mm}$ was considered and the inter-hole distances within the unit cell was $5 \mathrm{~mm}$. Calculated transmitted ultrasound power coefficient as a function of frequency, $f$, in water at normal incidence of plates with no vacancies and plates with one two or three vacancies in their unit cells, are showed separately in Figures 13 (a-d). When there are no vacancies in the unit cell (Fig. 13a), the resulting sample is a square lattice holes array with unit cell period $5 \mathrm{~mm}$. The transmission peak corresponds to the Fabry-Perot resonance, and the transmission dip at the frequency $296 \mathrm{kHz}$ correspond to the Wood anomaly for $(\mathrm{m}, \mathrm{n})=(1,0)$. The effect of 
a vacancy in the unit cell is showed in Figure 13b. In this case, the periodicity is broken and new transmission dips resulting from Wood anomaly corresponding to unit cell period of $15 \mathrm{~mm}$ appear. As could be expected, the amplitude of the Fabry-Perot resonance peak is reduced. As more vacancies are created in the unit cell (Fig. 13c-d), the transmission dips resulting from Wood anomaly remain invariable and there is a reduction in the amplitude of the Fabry-Perot resonance peak. Further investigation toward the effect of lattice vacancies in subwavelength perforated plates is needed.

\section{CONCLUSIONS}

The role of the geometrical parameters on the ultrasonic transmission through subwavelength holes arrays has been analized by using a theoretical model under the rigid-solid assumption. It has been pointed out that the transmission spectrum exhibit peaks because of the Fabry-Perot resonances and dips related to the Wood anomaly. The number and position of the transmission peaks depend on the plate thickness and they become narrower as the filling fractions decrease. The transmission dips corresponding to the Wood anomalies depend on the lattice period and geometry. When a plate is drilled with two periodic subwavelength holes arrays, the transmission spectrum is simply the addition of the primary and secondary array spectrum. Multiple-sublattice and fractalfeatured hole arrays have been analyzed. Prospective applications of the results presented in this paper can be anticipated, such as in the development of underwater sound screening materials, ultrasonic filters, ultrasonic medical instrumentation and non-destructive inspections.

\section{Acknowledgments}

This work has been supported by the Spanish MICINN (MAT2010-16879). 


\section{REFERENCES}

[1] L. Rayleigh: The Theory of Sound, vol. II, 2nd edn. Courier Dover Publications 1945.

[2] L. Brillouin: Wave Propagation in Periodic Structures. Dover, New York ,1953

[3] E. Yablonovitch: Inhibited spontaneous emission in solid state physics and electronics. Phys. Rev. Lett. 58, 2059, 1987.

[4] T.W. Ebbesen, H.J. Lezec, H.F.Ghaemi, T. Thio, P.A. Wolff: Extraordinary optical transmission through sub-wavelength hole arrays. Nature 39, 667-669, 1998.

[5] H.A. Bethe: Theory of diffraction by small holes. Phys. Rev. 66(7-8), 163-182 1944.

[6] F.J. Garcia-Vidal, L. Martin-Moreno, T.W. Ebbesen, L. Kuipers: Light passing through subwavelength apertures, Reviews of Modern Physics, 82, 729-787, 2010.

[7] M.H Lu, X.K. Liu, L. Feng, J. Li, C.P. Huang, Y.F. Chen, Y.Y. Zhu, S.N. Zhu, N.B. Ming: Extraordinary acoustic transmission through a 1d grating with very narrow apertures. Phys. Rev. Lett. 99(17), 174301,2007.

[8] L. Zhou, G.A. Kriegsmann: Complete transmission through a periodically perforated rigid slab. J. Acoust. Soc. Am. 121(6), 3288-3299, 2007.

[9] B. Hou, J. Mei, M. Ke, W. Wen, Z. Liu, J. Shi, P. Sheng: Tuning Fabry-Perot resonances via diffraction evanescent waves. Phys. Rev. B 76(5), 054303, 2007.

[10] J. Christensen, L. Martin-Moreno, F.J. Garcia-Vidal: Theory of resonant acoustic transmission through subwavelength apertures. Phys. Rev. Lett. 101(1), 014301, 2008. 
[11] H. Estrada, P. Candelas, A. Uris, F. Belmar, F.J. García de Abajo, F. Meseguer: Extraordinary sound screening in perforated plates. Phys. Rev. Lett. 101(8), 084302, 2008.

[12] R. W. Wood: Anomalous diffraction gratings. Phys. Rev. 48(12), 928-936, 1935.

[13] H. Estrada, F.J. García de Abajo, P. Candelas, A. Uris, F. Belmar, F. Meseguer: Angle dependent ultrasonic transmission through plates with subwavelength hole arrays. Phys. Rev. Lett. 102(14), 144301, 2009.

[14] X. Wang: Acoustical mechanism for the extraordinary sound transmission through subwavelength apertures. Appl. Phys. Lett. 96(13), 134104, 2010.

[15] X. Wang: Theory of resonant sound transmission through small apertures on periodically perforated slabs. J. Appl. Phys. 108(6), 064903, 2010.

[16] Z. Liu, G. Jin: Resonant acoustic transmission through compound subwavelength hole arrays: The role of phase resonances. J. Phys. Condens. Matter 21(44), 445401,2009.

[17] R. Hao, H. Jia, Y. Ye, F. Liu, C. Qiu, M. Ke, Z. Liu, Exotic acoustic transmission through hard plates perforated with quasiperiodic subwavelength apertures. Eur. Phys. Lett. 92, 24006, 2010.

[18] H. Estrada, P. Candelas, A. Uris, F. Belmar, F. Meseguer, F.J. García de Abajo: Influence of the hole filling fraction on the ultrasonic transmission through plates with subwavelength aperture arrays. Appl. Phys. Lett. 93(1), 011907, 2008.

[19] H. Estrada, P. Candelas, A. Uris, F. Belmar, F.J. García de Abajo, F. Meseguer: Influence of lattice symmetry on ultrasound transmission through plates with subwavelength aperture arrays. Appl. Phys. Lett. 95(5), 051906, 2009. 
[20] H. Estrada, V. Gómez-Lozano, A. Uris, P. Candelas, F. Belmar, F. Meseguer, Sound transmissión through plates perforated with two periodic subwavelength hole arrays, J. Phys.: Condens. Matter 23, 135401, 2011.

[21] H. Estrada, V. Gómez-Lozano, A. Uris, P. Candelas, F. Belmar, F. Meseguer, Ultrasonic transmissión through multiple sublattice subwavelength hole arrays, Ultrasonics 52, 412-416, 2012.

[22] V. Gómez-Lozano, A. Uris, P. Candelas, F. Belmar, Acoustic transmission through perforated plates with fractal subwavelength apertures, Solid State Communications165, 11-14, 2013.

[23] H. Estrada, P. Candelas, A. Uris, F. Belmar, F.J. Garcia de Abajo, F. Meseguer: Sound transmission through perforated plates with subwavelength hole arrays: a rigidsolid model, Wave Motion 48, 235-242, 2010

[24] C. Kittel: Introduction to Solid State Physics 7th ed. Wiley, New York, 1966.

[25] E. G. Williams: Fourier Acoustics: Sound Radiation and Nearfield Acoustical Holography, Academic Press, New York, 1999.

[26] D. H. Towne: Wave Phenomena, Dover, New York, 1967.

\section{FIGURE CAPTIONS}

Figure 1. Scheme of the (a) 'xz' plane and (b) 'xy' plane of the unit cell. Grey regions correspond to the rigid solid whereas the surrounding fluid is divided in three regions as indicated by the labels in (a). The vector $\left(\mathbf{r}-\mathbf{r}_{i}\right)_{\|}$in (b) represents the projection over the $\mathrm{z}=0$ plane of the vector defined from the centre of each hole to the appropriate points in the hole fluid region [II]. 
Figure 2. (a) Calculated transmitted ultrasound power coefficient for a plate drilled with square lattice subwavelength holes arrays of diameter $d=2 \mathrm{~mm}$, lattice period $\mathrm{p}=4 \mathrm{~mm}$ and plate thickness $\mathrm{t}=2 \mathrm{~mm}$ (a) as a function of frequency in water at normal incidence. (b) as a function of both the frequency and the incidence angle.

Figure 3. Calculated transmitted ultrasound power coefficient as a function of frequency, $f$, in water at normal incidence for a plate drilled with square lattice subwavelength holes arrays of diameter $d=2 \mathrm{~mm}$, lattice period $p=4 \mathrm{~mm}$ and for plate thickness (a) $t=2 \mathrm{~mm}$, (b) $\mathrm{t}=3 \mathrm{~mm}$, (c) $\mathrm{t}=4 \mathrm{~mm}$ and (d) $\mathrm{t}=5 \mathrm{~mm}$.

Figure 4. Calculated transmitted ultrasound power coefficient as a function of frequency, $f$, in water at normal incidence for a plate drilled with square lattice subwavelength holes arrays of diameter $d=3 \mathrm{~mm}$, plate thickness $t=4 \mathrm{~mm}$ and for three lattice periods $p=4,6$ and $8 \mathrm{~mm}$.

Figure 5. Calculated transmitted ultrasound power coefficient as a function of frequency, $f$, in water at normal incidence for a plate of thickness $\mathrm{t}=4 \mathrm{~mm}$, drilled with square lattice subwavelength holes arrays of lattice period $p=6 \mathrm{~mm}$ and for three holes diameters $d=2$, 3 and $4 \mathrm{~mm}$.

Figure 6. Comparison of calculated transmitted ultrasound power coefficient as a function of frequency, $f$, in water at normal incidence for a plate arranged with square and triangular lattice hole arrays. The plate thickness was $\mathrm{t}=4 \mathrm{~mm}$, hole diameter $\mathrm{d}=2 \mathrm{~mm}$ and for lattice periods (a) $p=4 \mathrm{~mm}$, (b) $p=5 \mathrm{~mm}$ and (c) $p=6 \mathrm{~mm}$. 
Figure 7. Schematic diagram of the compound hole array considered.

Figure 8. Calculated transmitted ultrasound power coefficient as a function of frequency, $f$, for (a) the primary array and (b) the secondary array.

Figure 9. Schematic diagram of the multiple-sublattice hole unit cells.

Figure 10. Calculated transmitted ultrasound power coefficient as a function of frequency, $f$, in water at normal incidence of $4 \times 4$ multiple-sublattice holes arrays. The plate thickness is $t=3 \mathrm{~mm}$, the period of the unit cell is $\mathrm{a}=5 \mathrm{~mm}$, the hole diameter $\mathrm{d}=0.7$ and the inter-hole distance (a) $\mathrm{p}=\mathrm{a} / 4$, (b) $\mathrm{p}=\mathrm{a} / 5$ and (c) $\mathrm{p}=\mathrm{a} / 6$.

Figure 11. Schematic diagram of the iterative generations of the Sierpinski Carpet pattern. (a) First iteration with circular holes of diameter $0.5 \mathrm{~mm}$ and unit cell period 1.5 mm. (b) Second one with circular holes of diameters $0.5 \mathrm{~mm}$ and $1.5 \mathrm{~mm}$ and unit cell period $4.5 \mathrm{~mm}$. (c) Third iterative generation with circular holes of diameters $0.5 \mathrm{~mm}$, $1.5 \mathrm{~mm}$ and $4.5 \mathrm{~mm}$ and unit cell period $13.5 \mathrm{~mm}$.

Figure 12. Calculated transmitted ultrasound power coefficient as a function of frequency, $f$, in water at normal incidence for (a) First iteration with circular holes of diameter 0.5 $\mathrm{mm}$ and unit cell period $1.5 \mathrm{~mm}$. (b) Second one with circular holes of diameters 0.5 $\mathrm{mm}$ and $1.5 \mathrm{~mm}$ and unit cell period $4.5 \mathrm{~mm}$. (c) Third iterative generation with circular holes of diameters $0.5 \mathrm{~mm}, 1.5 \mathrm{~mm}$ and $4.5 \mathrm{~mm}$ and unit cell period $13.5 \mathrm{~mm}$. 
Figure 13. Calculated transmitted ultrasound power coefficient as a function of frequency, $f$, in water at normal incidence of $3 \times 3$ squared holes arrays. The holes diameter was $3 \mathrm{~mm}$ and the plate thickness $2 \mathrm{~mm}$. A unit cell period of $15 \mathrm{~mm}$ was considered and the inter-hole distances within the unit cell was $5 \mathrm{~mm}$. (a) No vacancies in the unit cell. (b) One vacancy in the unit cell. (c) Two vacancies in the unit cell. (d) Three vacancies in the unit cell. 


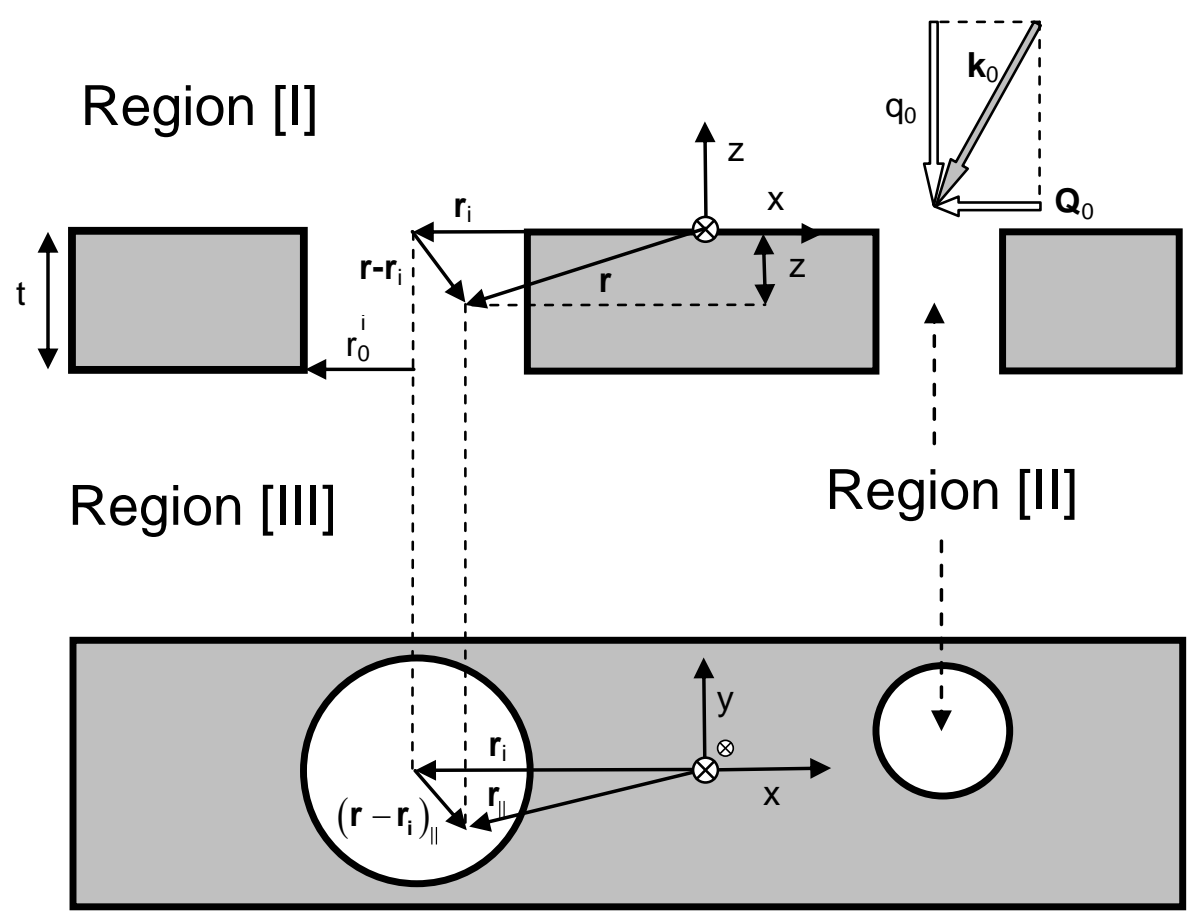

(a)

(b) 

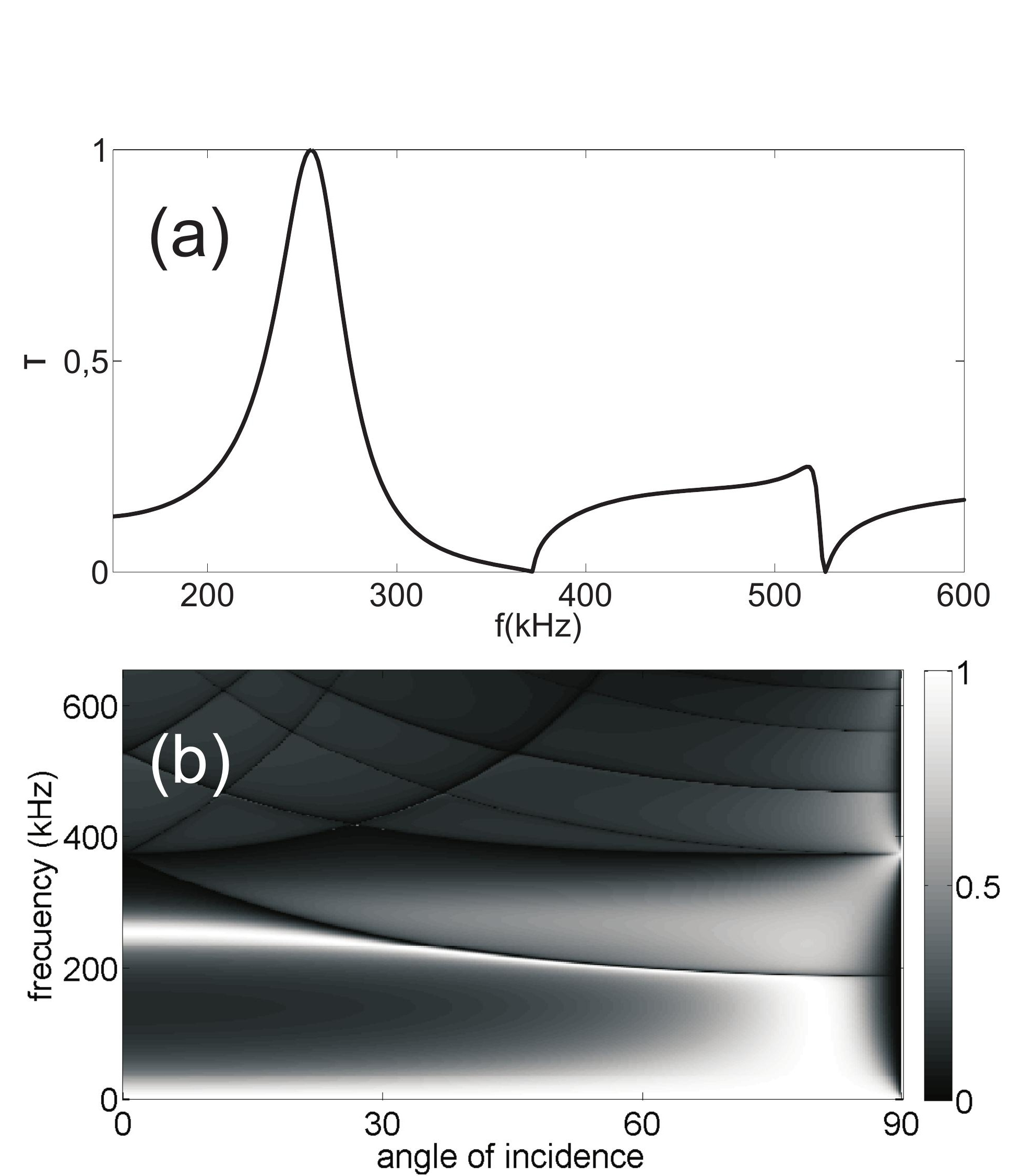

.



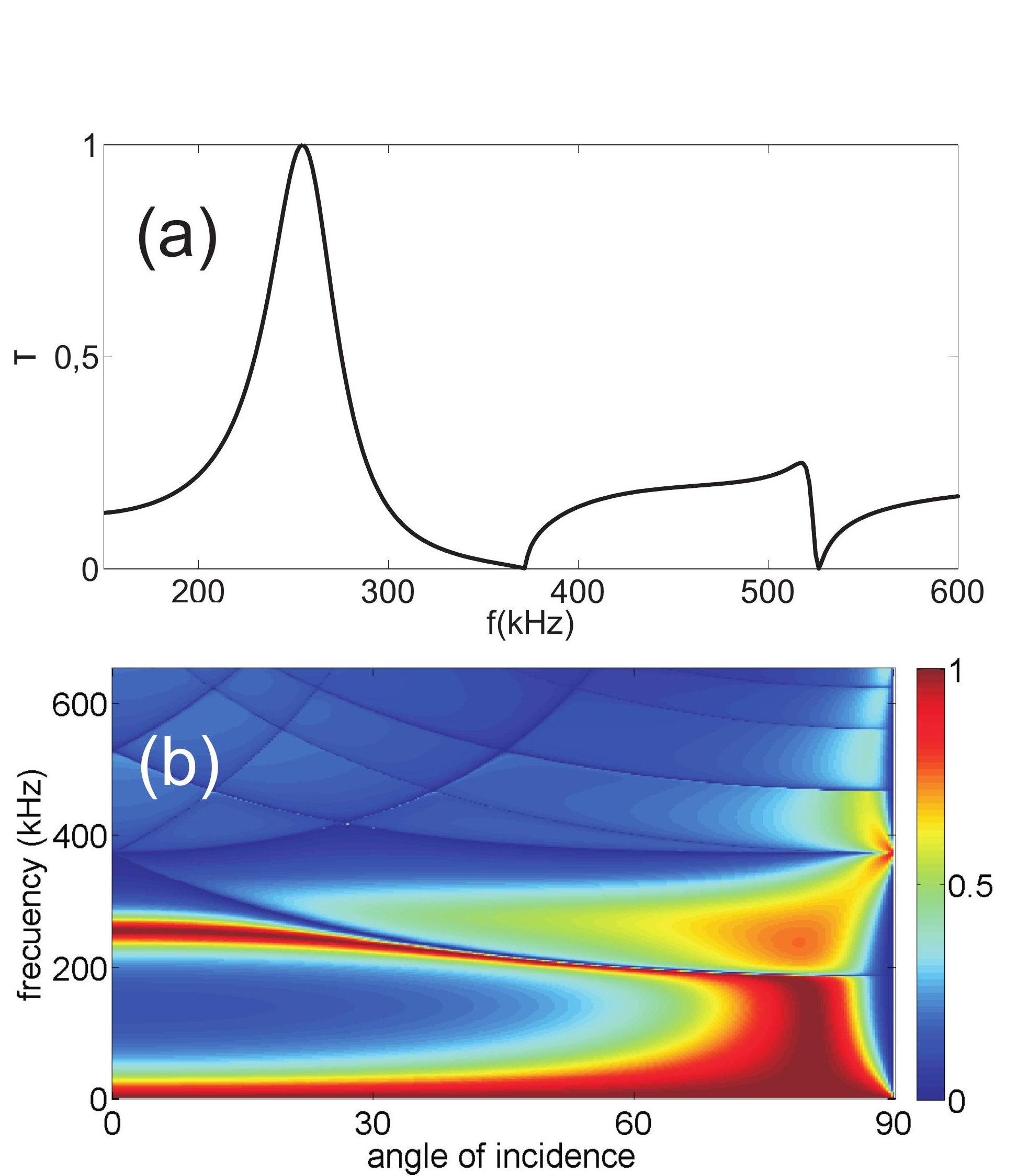
Figure 3
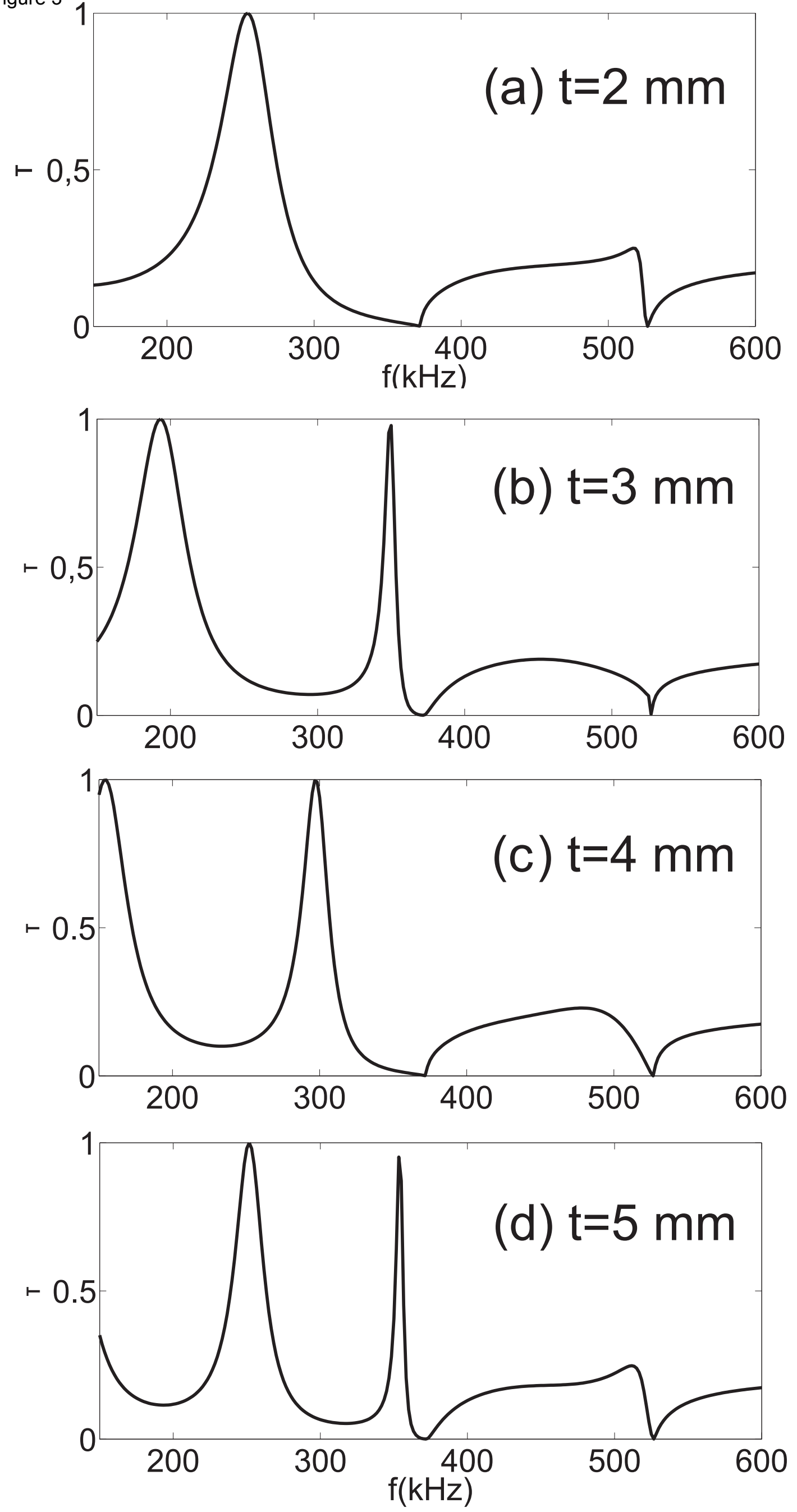
Figure 4

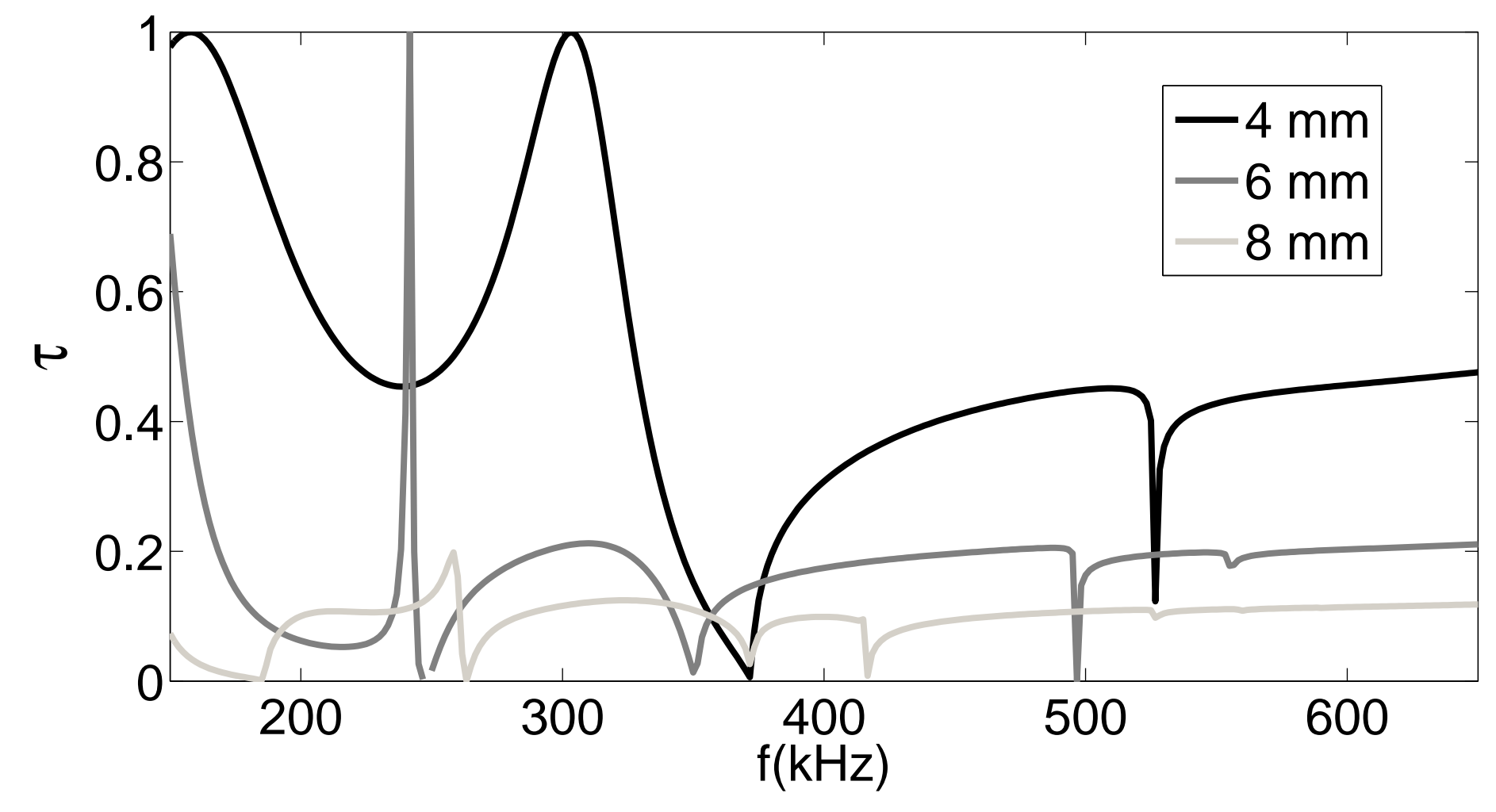

$\mathrm{f}(\mathrm{kHz})$ 


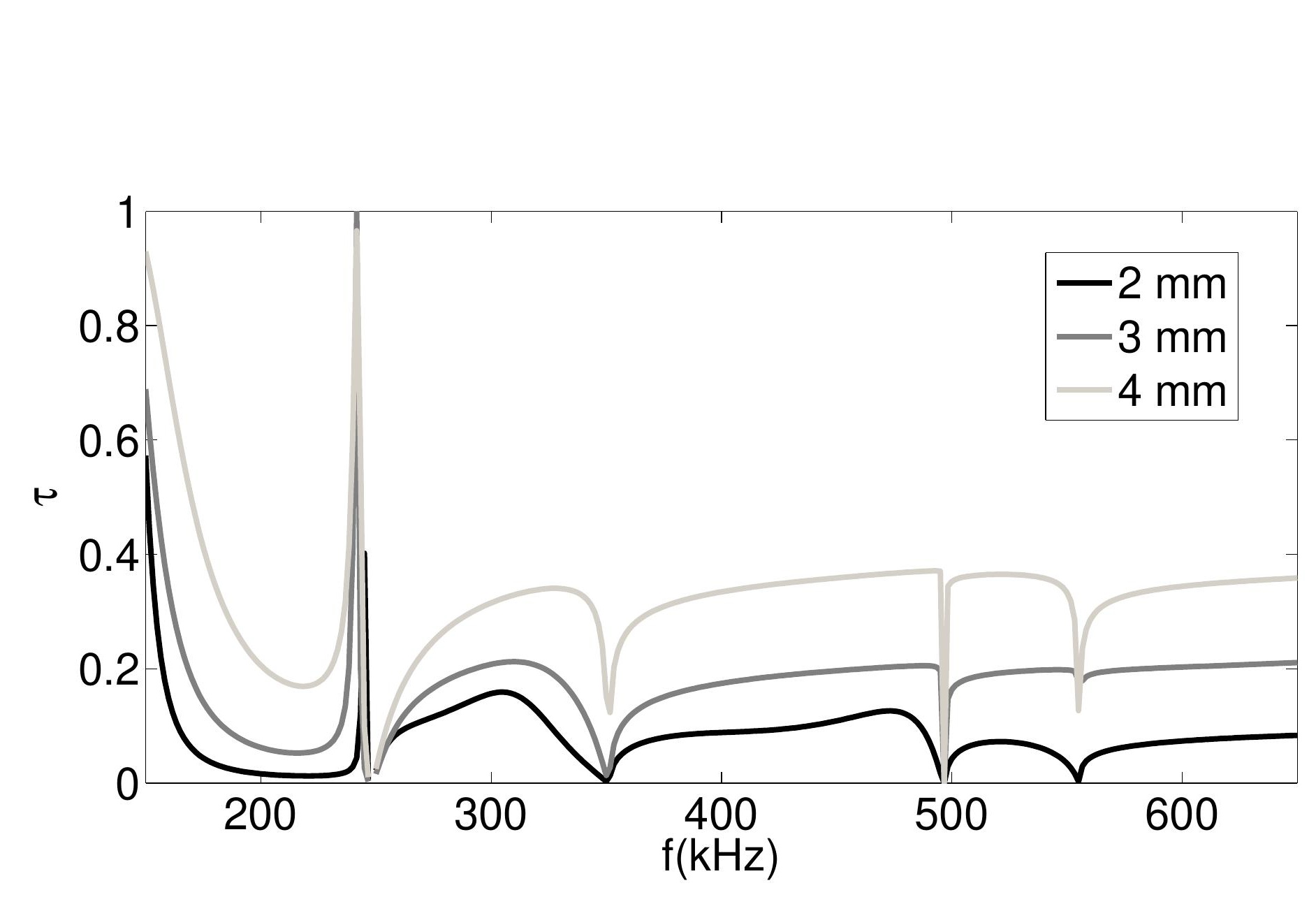

Figure 5

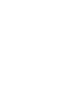

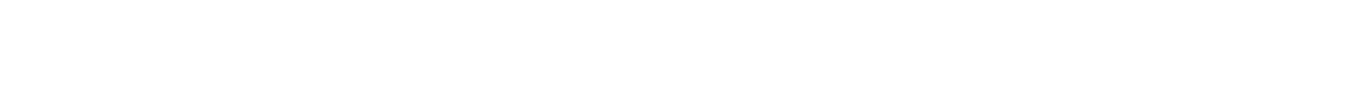

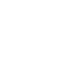

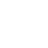

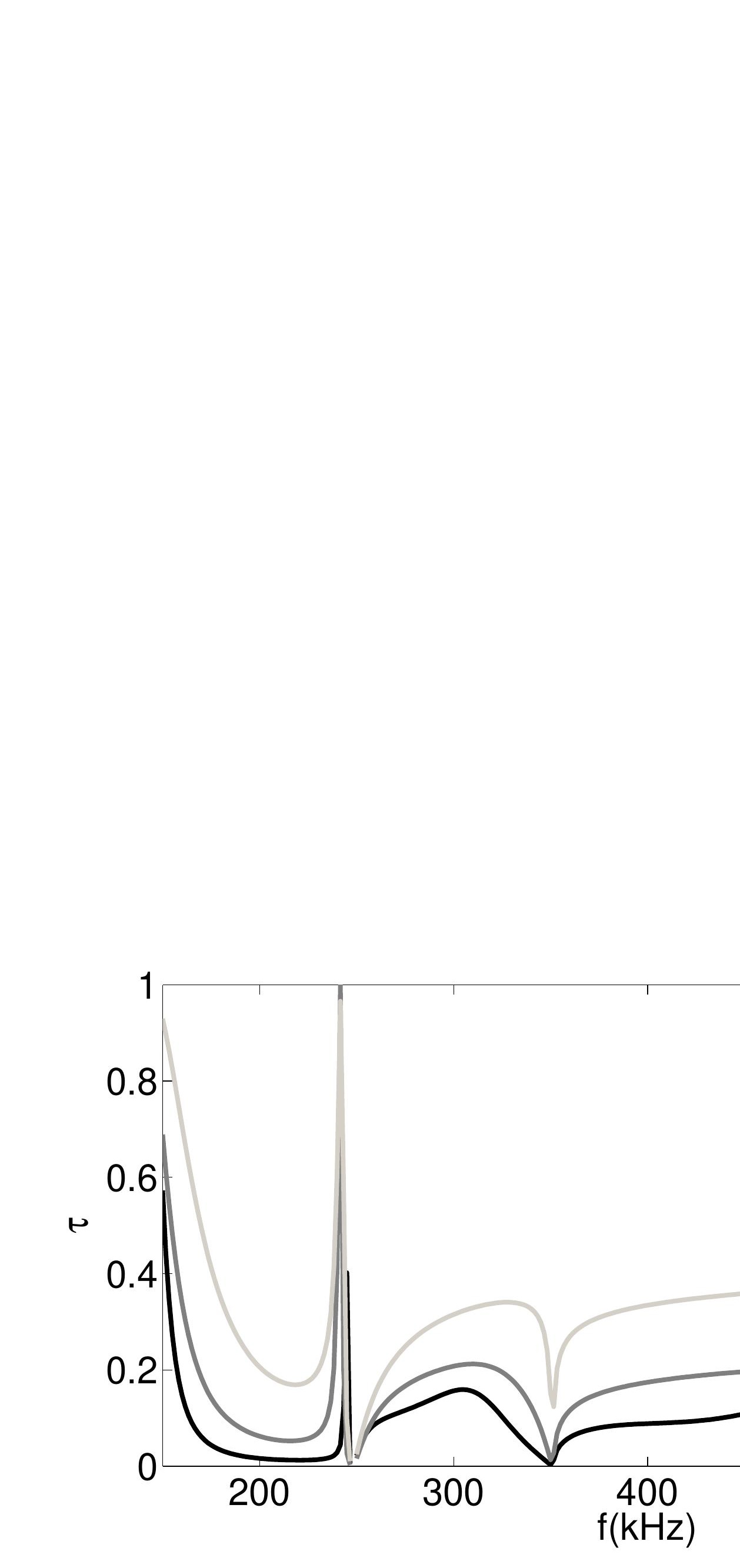

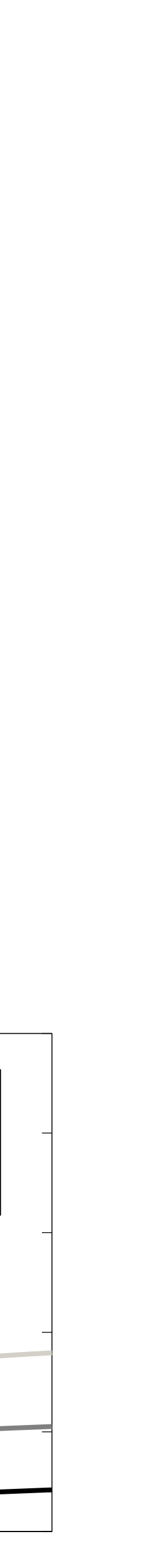



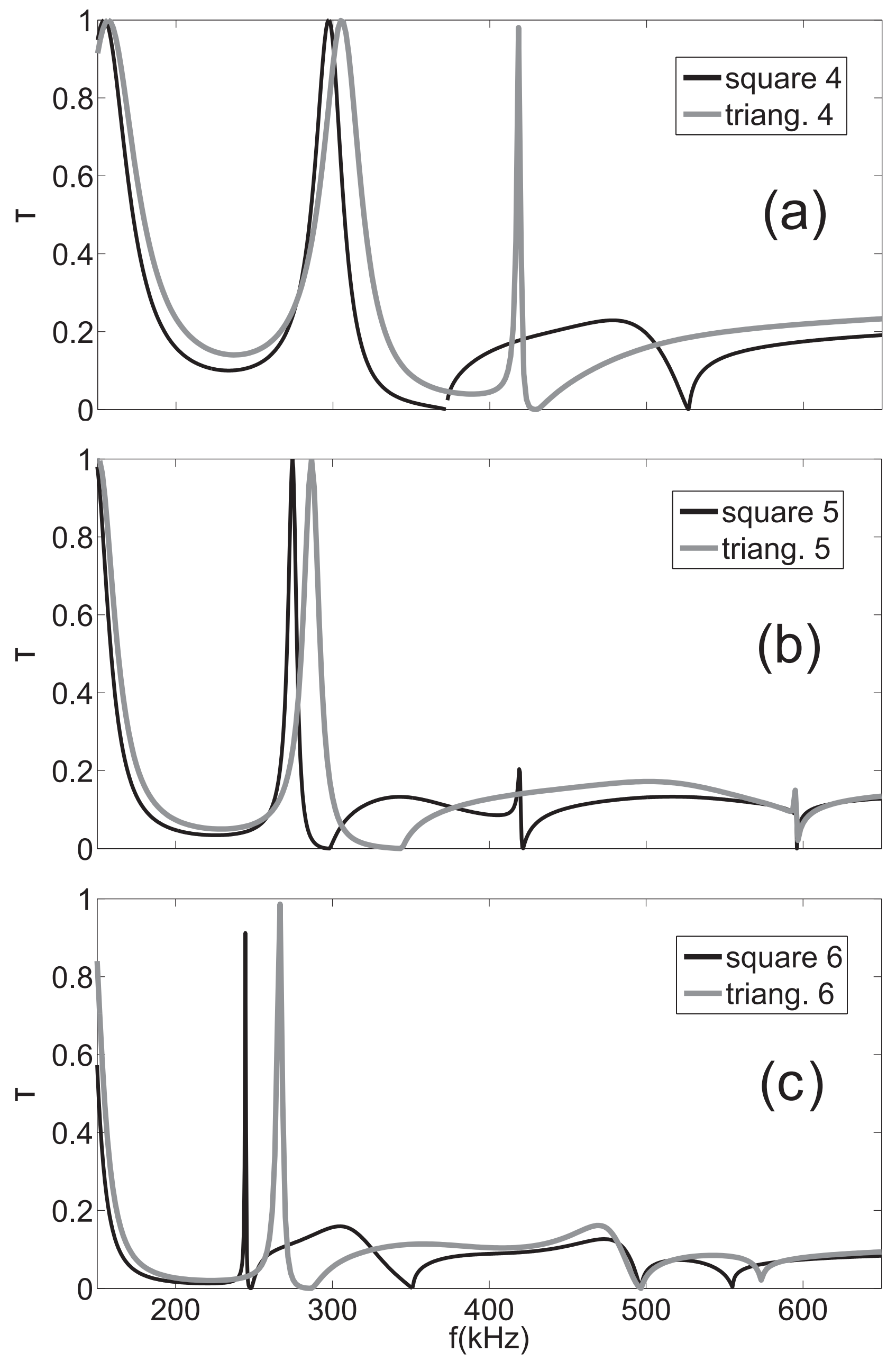


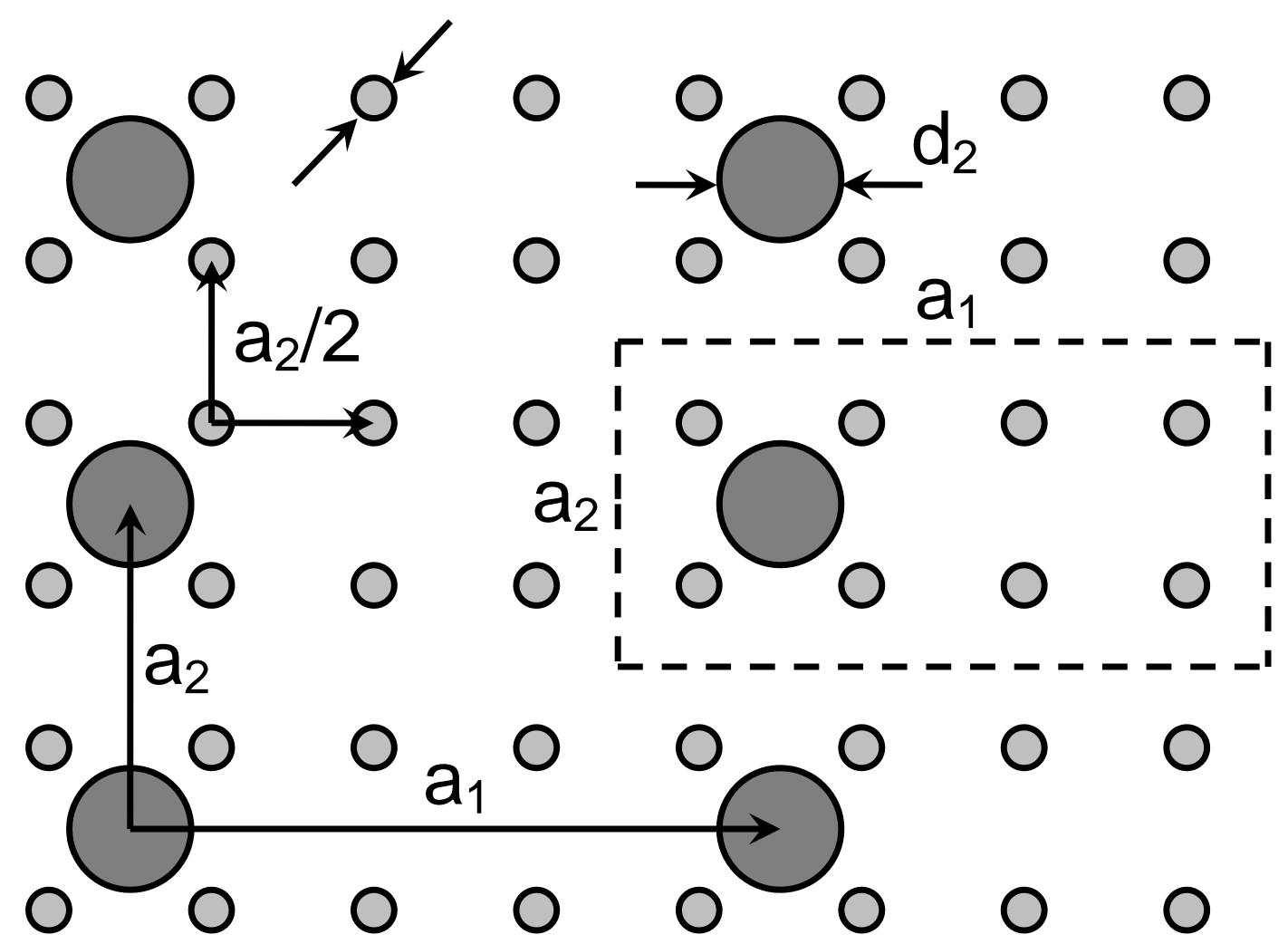


Figure 8
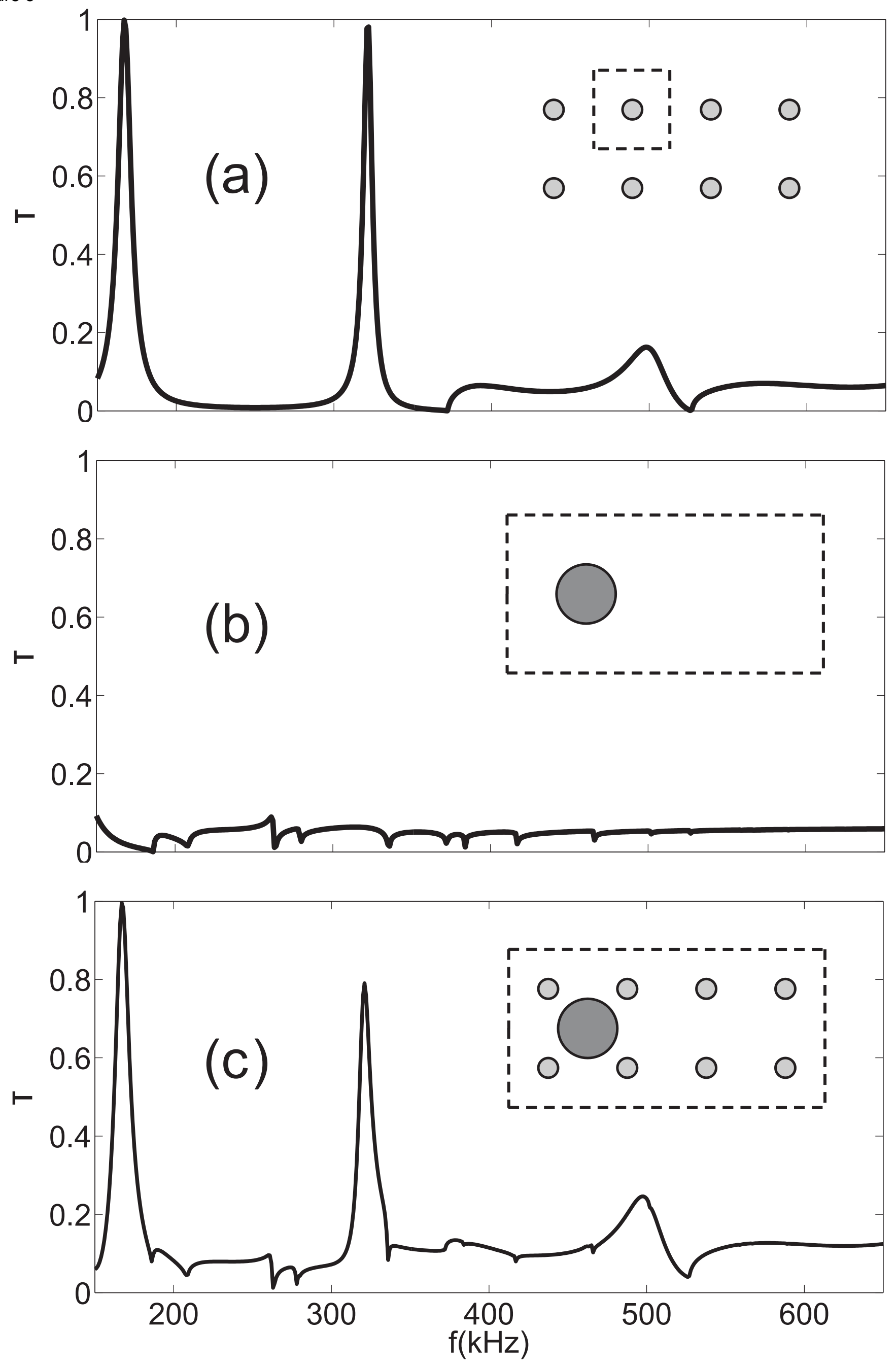

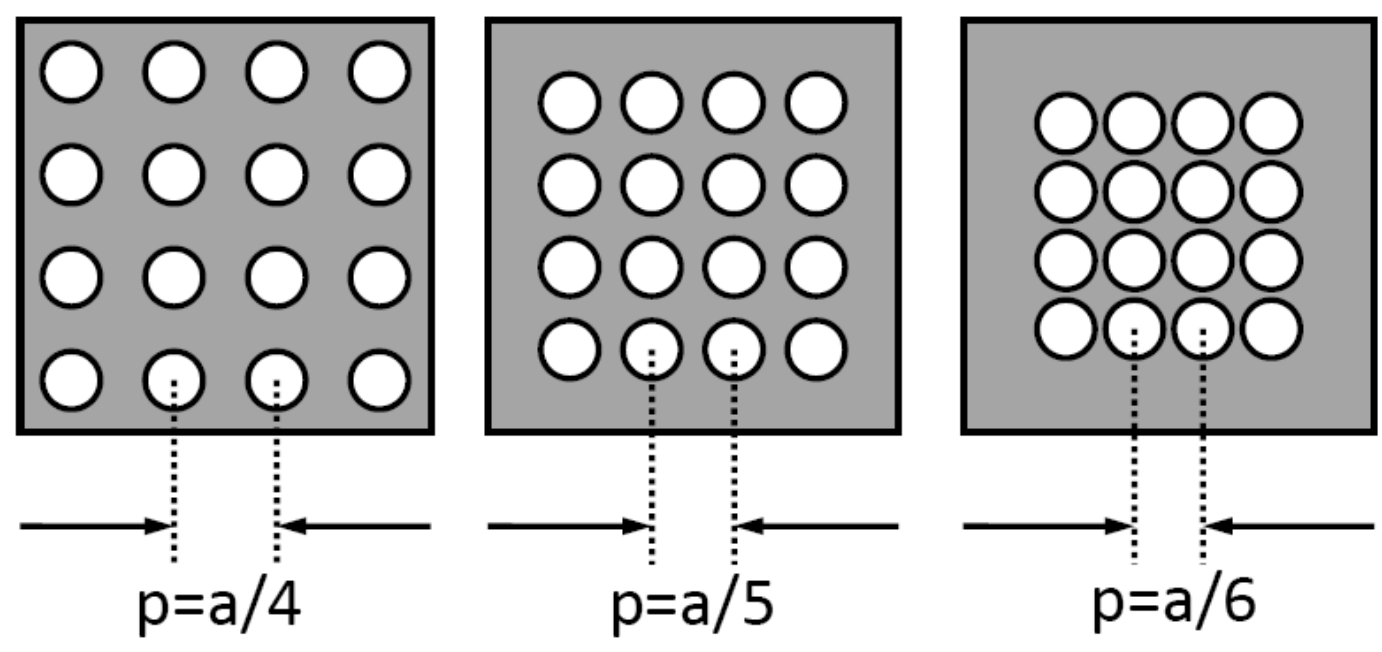
Figure 10
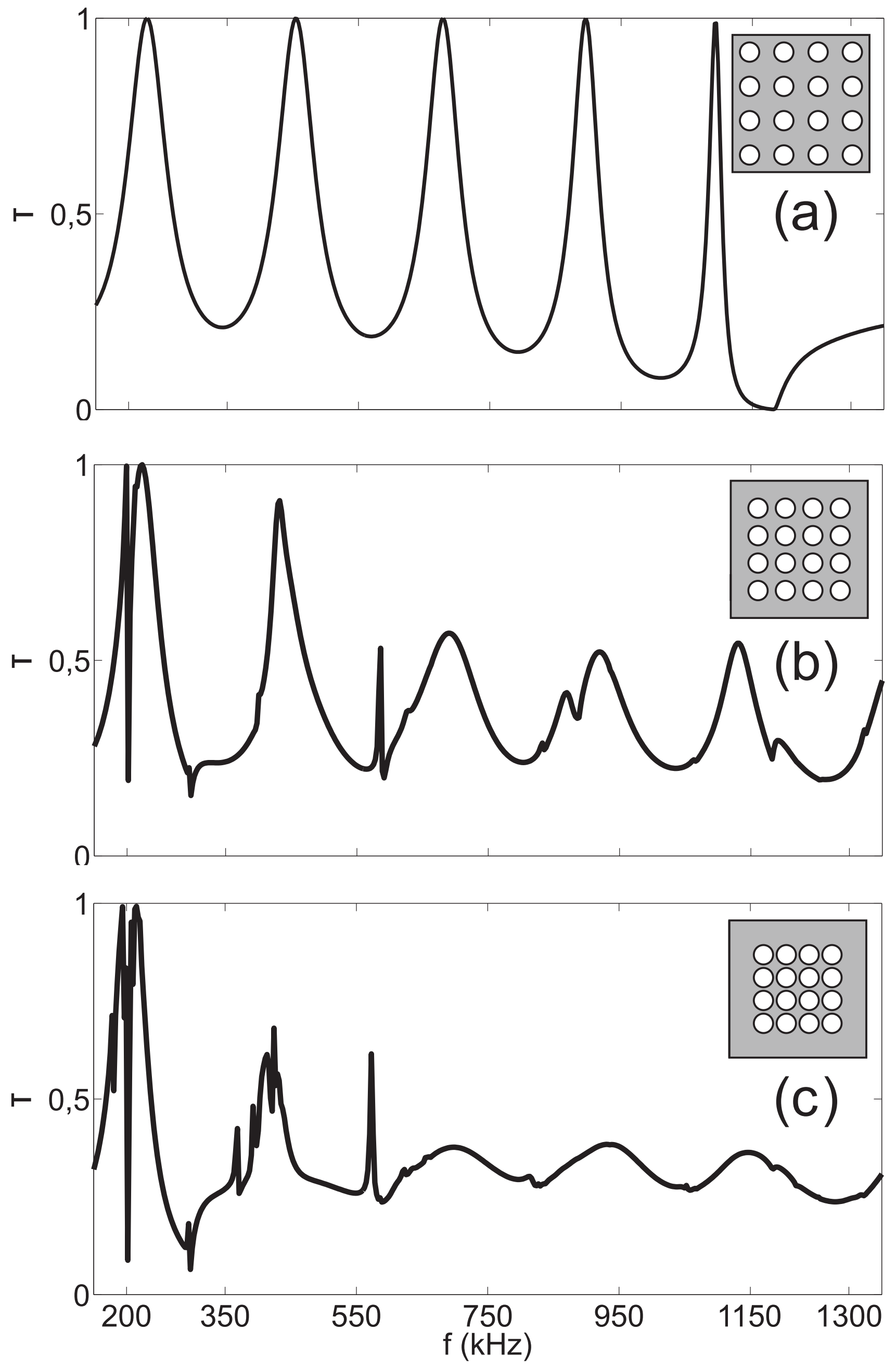


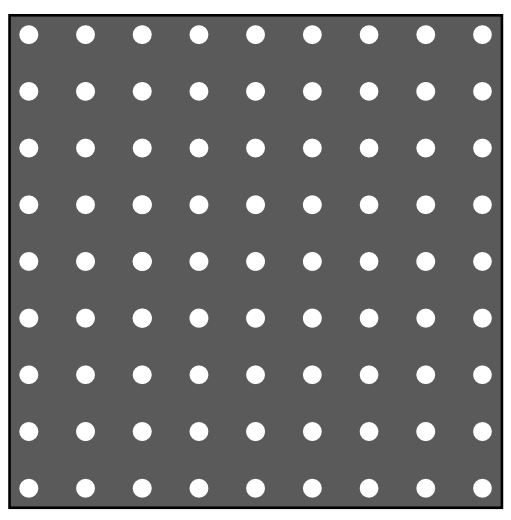

(a)

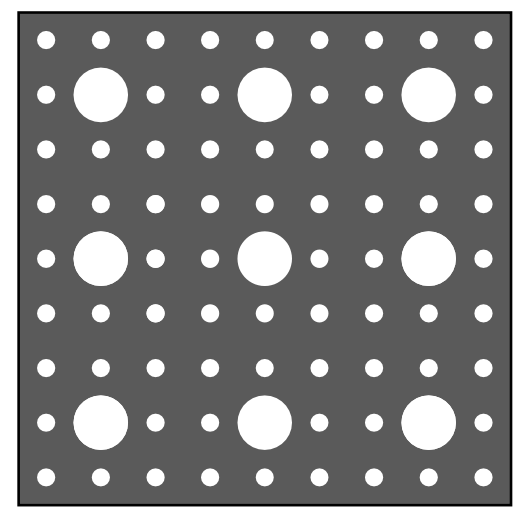

(b)

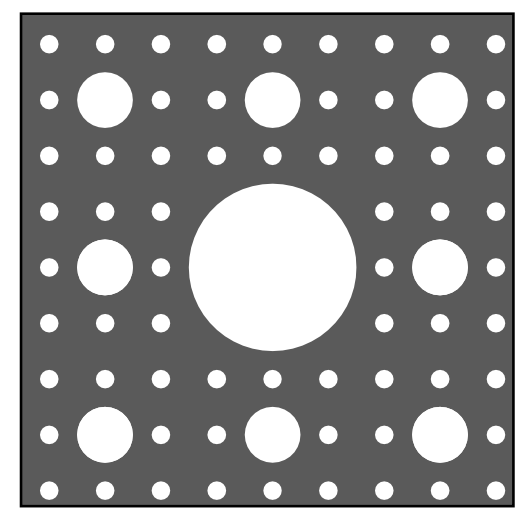

(c) 
Figure 12
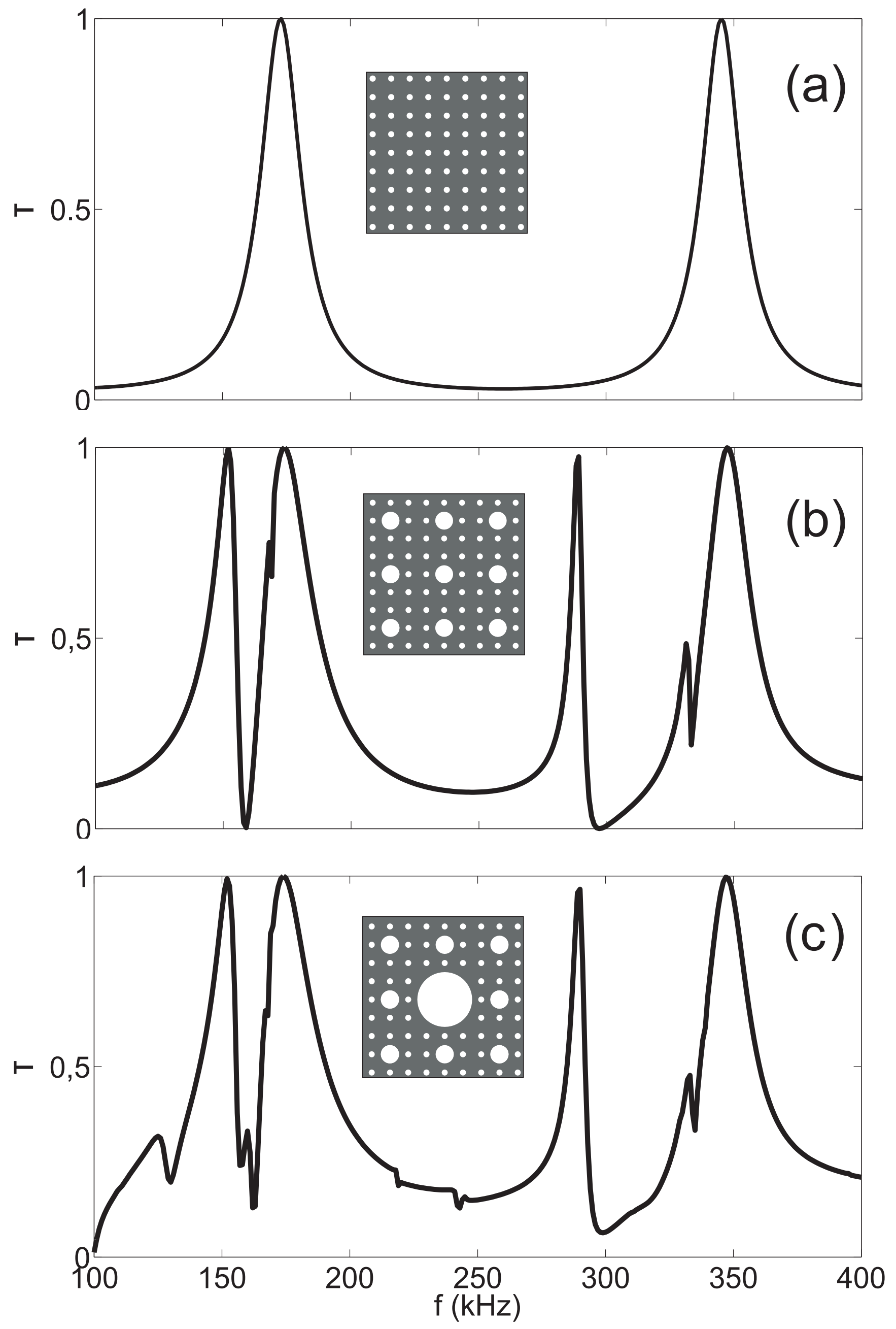
Figure 13
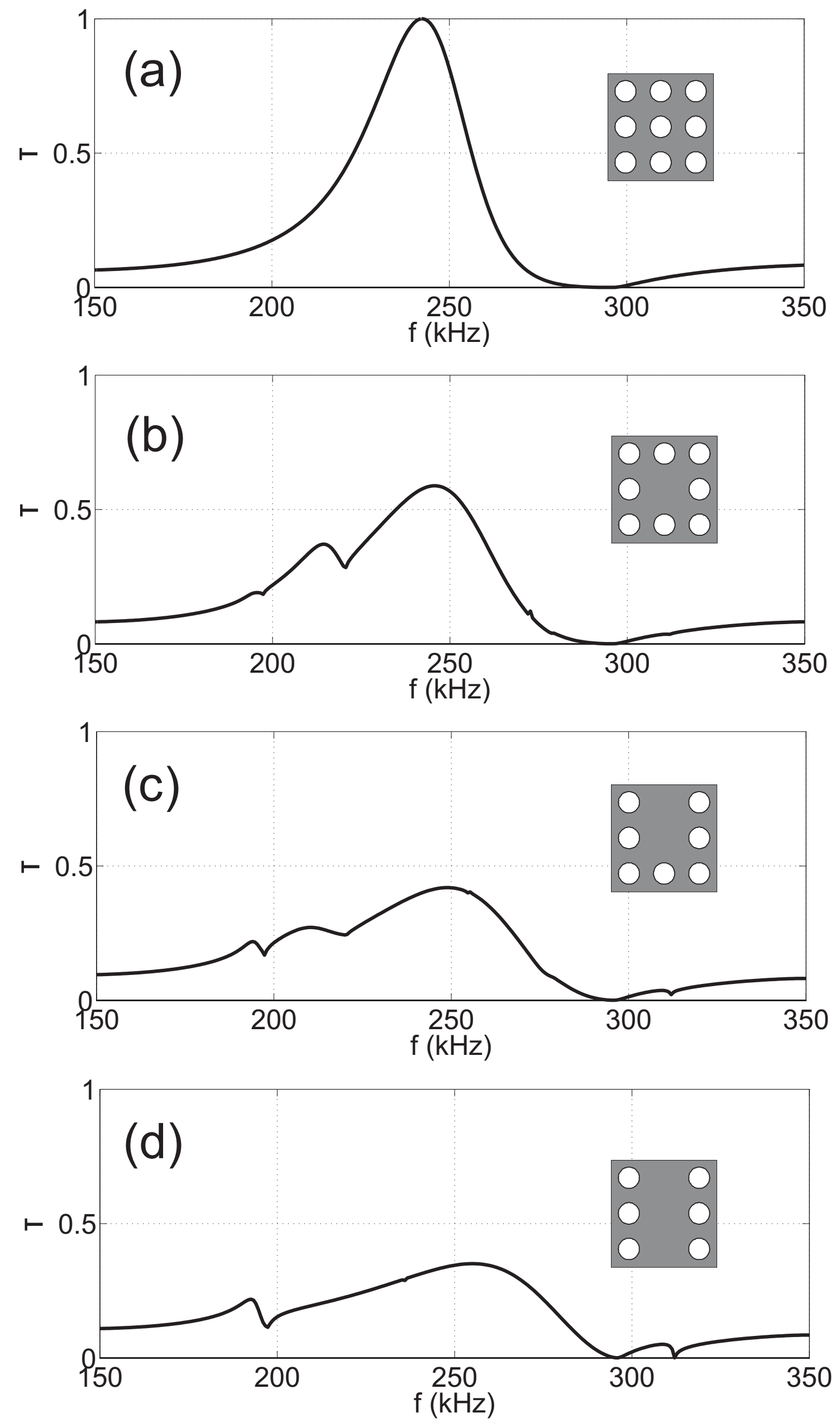